\section{Ekzemschübe durch Kreuzreaktionen}

\section{Es ist bekannt, dass bei Kindern mit atopischem Ekzem Nahrungs- mittel wie Kuhmilch, Eier oder Sojaprodukte zu Ekzemschüben führen können. Pollen-assoziierte Nahrungsmittelallergien als Aus- löser standen dagegen bisher weniger im Fokus.}

B irkenpollen-assoziierte Nahrungsmittel stammen vor allem aus den Pflanzenfamilien der Rosaceae, Solanaceae und Umbelliferae. Mit den Vertretern Apfel, Haselnuss, Karotte oder Sellerie zählen sie zur Basisernährung und können außer Sofortreaktionen auch ekzematöse Spätreaktionen 6-48 Stunden nach Nahrungsaufnahme induzieren.

An zwölf Kindern mit einem mittleren Alter von 5 Jahren wurde dies überprüft. Alle Kinder litten unter mittelschwerem bis schwerem atopischen Ekzem und wiesen Birkenpollen-spezifisches $\operatorname{IgE}$ auf. Bisher hatten die Kinder keine Sofortreaktionen auf die zur Provokation verwendeten Birkenpollen-assoziierten Nahrungsmittel, die sie regelmäßig verzehrten, gezeigt. Ein Teil der Kinder litt unter Atemwegssymptomen während der Birkenpollensaison oder reagierte in dieser Zeit mit Hautverschlechterungen. Alle wurden einer doppelblinden, plazebokontrollierten Nahrungsmittelprovokation unterzogen.

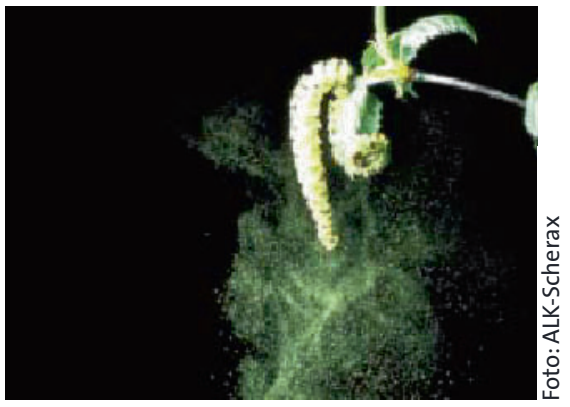

Birkenpollen-assoziierte Nahrungsmittel scheinen als Auslöser von Ekzemschüben bei Kindern eine Rolle zu spielen.
Sieben der zwölf Kinder reagierten mit einer allergischen Früh- und/oder Spätreaktion, vier zeigten eine ekzematöse Spätreaktion innerhalb von 24 Stunden mit einer signifikanten Zunahme des SCORAD-Index. Die Verschlechterung manifestierte sich meist als „Aufblühen“ bereits vorhandener ekzematöser Läsionen. Die vier Kinder unterschieden sich weder in Anamnese noch spezifischem IgE von den Kindern ohne Reaktion, bei drei der positiv reagierenden Kinder fand sich im CAP-RAST auch spezifisches IgE gegen die Birkenpollen-assoziierten Nahrungsmittel.

Fazit: Bei Kindern mit schwerem atopischen Ekzem und Birkenpollen-spezifischem $\operatorname{IgE}$ sollte auch ohne Vorliegen eines Verdachts eine Provokation mit Birkenpollen-assoziierten Nahrungsmitteln erwogen werden.

Breuer K et al. Birch pollen-related food as a provocation factor of allergic symptoms in children with atopic eczema/dermatitis syndrome. Allergy 2004; 59: 988-94

\title{
Kombitherapie bei Urtikaria ohne Vorteil
}

\section{Mittel der ersten Wahl bei einer chronischen idiopathischen Urtikaria (CIU) sind $\mathrm{H}_{1}$-Blocker. Es liegen aber auch Berichte über Therapieerfolge mit Leukotrienrezeptor-Antagonisten (LT-RA) vor. Größere systematische Untersuchungen zum Einsatz von LT-RA bei Urtikaria gibt es aber noch nicht.}

n einer randomisierten, doppelblind
mit der Double-dummy-Technik
durchgeführten, plazebokontrollierten
Vergleichsstudie erhielten 160 Patienten
mit einer mittelschweren CIU 6 Wo-
chen lang 5 mg des H1-Blockers Des-
loratadin einmal täglich ( $=40), 10$ mg
des LT-RA Montelukast einmal täglich
(n $=40), 5$ mg Desloratadin morgens
plus 10 mg Montelukast abends (n = 40)
oder ausschließlich Plazebo ( $=40$ ).
Ausgeschlossen waren Patienten mit
urtikariellen Symptomen nach Provo-
kation durch Acetylsalicylsäure, andere
nicht-steroidale Antiphlogistika oder
Nahrungsmittelzusätze sowie Patienten mit einer physikalischen oder allergisch bzw. autoimmunologisch bedingten Urtikaria.

Nur die Patientengruppen mit der Desloratadin-Monotherapie sowie der Desloratadin-Montelukast-Kombinationstherapie konnten über die gesamte Studiendauer verfolgt werden. Unter der Montelukast-Monotherapie beendeten 27 der 40 Patienten und in der Plazebogruppe 35 der 40 Patienten die Studie vorzeitig. Bei der Auswertung der Parameter Gesamtsymptomscore, Anzahl der Quaddeln und Umfang der größten Quaddel ergaben sich bei allen Verumgruppen signifikante Unterschiede zu Plazebo. Beim
Symptom Juckreiz waren nur in der H1Blocker-Gruppe sowie in der Kombinationsgruppe signifikante Unterschiede zu Plazebo festzustellen, Montelukast hatte auf dieses Symptom keinen Einfluss. Unterschiede in der Wirksamkeit zwischen Desloratadin als Monotherapie und Desloratadin in Kombination mit Montelukast ergaben sich nicht.

Fazit: Bei Patienten mit einer CIU bietet der H1-Blocker Desloratadin eine gute Therapiemöglichkeit zur Bekämpfung von Hautsymptomen und Juckreiz. Die zusätzliche Gabe des Leukotrienrezeptor-Antagonisten Montelukast bringt bei diesen Patienten keinen weiteren Vorteil.

$b k$

Di Lorenzo G et al. Randomized placebocontrolled trial comparing desloratadine and montelukast in monotherapy and desloratadine plus montelukast in combined therapy for chronic idiopathic urticaria. J Allergy Clin Immunol 2004; 114: 619-25 